\title{
Synthesis of Gold Nanorod/Single-Wall Carbon Nanotube Heterojunctions Directly on Surfaces
}

\author{
Aneta J. Mieszawska, Romaneh Jalilian, Gamini U. Sumanasekera, and Francis P. Zamborini
}

\section{Supporting Information}

Full Details of Synthesis of Au NR/SWCNT heterojunctions:

Au NR/SWCNT heterojunctions were synthesized directly on $\mathrm{Si} / \mathrm{SiOx}$ substrates as shown in Scheme 1

of the paper. First, an $\mathrm{Si} / \mathrm{SiO}_{\mathrm{x}}$ substrate was functionalized with aminopropyltriethoxysilane (APTES) by cleaning in 3:1 $\mathrm{H}_{2} \mathrm{SO} 4: \mathrm{H}_{2} \mathrm{O}_{2}$ (piranha) solution (Caution: piranha reacts violently with organic compounds), rinsing thoroughly with water and isopropanol (IPA), and then placing into a solution containing $100 \mu \mathrm{L}$ APTES, $10 \mathrm{~mL}$ IPA, and 2-3 drops of water while heating just below boiling for $30 \mathrm{~min}$. The substrate was removed, rinsed thoroughly with IPA, dried under $\mathrm{N}_{2}$, and placed into a very dilute dimethylformamide (DMF) solution of SWCNTs ${ }^{*}$ for 25-30 min. The substrate was removed, rinsed with DMF and IPA, and dried under $\mathrm{N}_{2}$. This leads to a low coverage of SWCNTs adsorbed to the $\mathrm{NH}_{2}$ groups on the $\mathrm{Si} / \mathrm{SiOx} / \mathrm{APTES}$ substrate in the form of bundles containing 7-10 nanotubes. $1.6 \mathrm{~nm}$ average diameter hexanethiolate Au monolayer-protected clusters (MPCs) were prepared by the Brust method ${ }^{5}$ and physisorbed onto the surface-attached SWCNTs, presumably through hydrophobic interactions, by simply immersing the $\mathrm{Si} / \mathrm{SiOx} / \mathrm{APTES} / \mathrm{SWCNT}$ substrate into a $9 \mathrm{mg} / \mathrm{mL}$ toluene solution of the MPCs for 10-15 min. The substrate was rinsed with toluene and IPA, dried under $\mathrm{N}_{2}$, and placed into a "growth solution" containing $9 \mathrm{~mL} 0.1 \mathrm{M}$ cetyltrimethylammonium bromide (CTAB), $450 \mu \mathrm{L} 0.01 \mathrm{M} \mathrm{HAuCl4}$, and $50 \mu \mathrm{L}$ $0.01 \mathrm{M}$ ascorbic acid for $1 \mathrm{~h}$. This leads to the seed-mediated growth of the SWCNT-bound Au MPCs into $\mathrm{Au} \mathrm{NRs}$ and other shapes as described previously for $\mathrm{Au}$ NRs grown from citrate-stabilized Au nanoparticles in solution ${ }^{6 a}$ or attached to surfaces. ${ }^{6 b-d}$

* SWCNTs were synthesized by pulsed laser vaporization (YAG laser; 1064/532 nm) of a target containing graphite $/ \mathrm{Ni} / \mathrm{Co} / \mathrm{Y}$ in an argon atmosphere at $1200{ }^{\circ} \mathrm{C}$. Material was purified by oxidation in dry air at 400 ${ }^{\circ} \mathrm{C}$ followed by acid refluxing in $4 \mathrm{M} \mathrm{HNO} 3$ and high temperature annealing in vacuum.

References 5 and 6 are cited in the main paper. 